\title{
A Method to Solve the Limitations in Drawing External Rays of the Mandelbrot Set
}

\author{
M. Romera, ${ }^{1}$ G. Pastor, ${ }^{1}$ A. B. Orue, ${ }^{1}$ A. Martin, ${ }^{1}$ M.-F. Danca, ${ }^{2,3}$ and F. Montoya ${ }^{1}$ \\ ${ }^{1}$ Instituto de Tecnologías Físicas y de la Información (ITEFI), Consejo Superior de Investigaciones Científicas (CSIC), \\ Serrano 144, 28006 Madrid, Spain \\ ${ }^{2}$ Department of Mathematics and Computer Science, "Avram Iancu" University, Ilie Măcelaru 1A, 400380 Cluj-Napoca, Romania \\ ${ }^{3}$ Romanian Institute of Science and Technology, Cireşilor 29, 400487 Cluj-Napoca, Romania
}

Correspondence should be addressed to M. Romera; miguel@iec.csic.es

Received 9 July 2013; Accepted 4 August 2013

Academic Editor: Guanrong (Ron) Chen

Copyright (C) 2013 M. Romera et al. This is an open access article distributed under the Creative Commons Attribution License, which permits unrestricted use, distribution, and reproduction in any medium, provided the original work is properly cited.

\begin{abstract}
The external rays of the Mandelbrot set are a valuable graphic tool in order to study this set. They are drawn using computer programs starting from the Böttcher coordinate. However, the drawing of an external ray cannot be completed because it reaches a point from which the drawing tool cannot continue drawing. This point is influenced by the resolution of the standard for floatingpoint computation used by the drawing program. The IEEE 754 Standard for Floating-Point Arithmetic is the most widely used standard for floating-point computation, and we analyze the possibilities of the quadruple 128 bits format of the current IEEE 7542008 Standard in order to draw external rays. When the drawing is not possible, due to a lack of resolution of this standard, we introduce a method to draw external rays based on the escape lines and Bézier curves.
\end{abstract}

\section{Introduction}

As is well known, the Mandelbrot set can be defined by $\mathscr{M}=$ $\left\{c \in \mathbb{C}: f_{c}^{o k}(0) \nrightarrow \infty\right.$ as $\left.k \rightarrow \infty\right\}$, where $f_{c}^{o k}(0)$ is the $k$-iteration of the parameter-dependent quadratic function $f_{c}(z)=z^{2}+c(z$ and $c$ complex $)$ from the initial value $z_{0}=0$ (the critical point).

In the 1980s, Douady and Hubbard published the external arguments theory of the Mandelbrot set $[1,2]$. Douady popularized this theory [3] by considering a capacitor made of a hollow metallic cylinder with great diameter, whose axis was an aluminum bar shaped in such a way that its cross-section was $\mathscr{M}$. If the capacitor is connected to a battery an electric field appears between the cylinder and $\mathscr{M}$, with equipotential lines and field lines. The field lines are the external rays of Douady and Hubbard, and the numbers associated with the external rays (between 0 and 1) are the external arguments of Douady and Hubbard. This electric field is extremely complicated because it is generated in a capacitor where one of their plates is a fractal. For this reason, mathematicians and engineers may be interested in that study. The external rays and its external arguments identify graphically and numerically all the periodic components and Misiurewicz points (preperiodic points) of $\mathscr{M}$, and, therefore, the drawing of the external rays is important to study the ordering of $\mathscr{M}$.

The computer programs to draw external rays of the Mandelbrot set use the Böttcher coordinate $\Phi(c)$ given by [4]

$$
\Phi(c)=c \prod_{n=1}^{\infty}\left[1+\frac{c}{\left[f_{c}^{n-1}(c)\right]^{2}}\right]^{1 / 2^{n}}
$$

where $c$ is the complex coordinate of a point outside $\mathscr{M}$ and $f_{c}^{0}(c)=c, f_{c}^{1}(c)=c^{2}+c, f_{c}^{2}(c)=\left(c^{2}+c\right)^{2}+c \cdots$ are the iterates of $f_{c}(z)=z^{2}+c$ from the initial value $z_{0}=0$. The potential of $c$ is $\log |\Phi(c)|$, and the external argument of $c$ is $\arg \Phi(c)$ [2]. All the points of an external ray have the same external argument. All the points with the same potential define an equipotential line. The external rays are perpendicular to the equipotential lines. 
TABLE 1: Basic formats in the IEEE 754-2008 Standard [5].

\begin{tabular}{lccc}
\hline & Sign & Mantissa & Exponent \\
\hline Single (32 bits) & 1 & 23 & 8 \\
Double (64 bits) & 1 & 52 & 11 \\
Quadruple (128 bits) & 1 & 112 & 15 \\
\hline
\end{tabular}

The IEEE 754 Standard for Floating-Point Arithmetic [5] is the most widely used standard for floating-point computation, and it is followed by many hardware and software implementations. Many computer languages allow or require that some or all arithmetic be carried out using IEEE 754 formats and operations. The current version is IEEE 754-2008 that includes the original IEEE 754-1985. The resolutions of the three basic formats of this standard are res single $=23$, res $_{\text {double }}=52$ and res quadruple $=112$, according to the number of bits of the mantissas (see Table 1).

As we will see, the drawing of an external ray inside a detail of $\mathscr{M}$ is strongly restricted by the number of bits of the floating-point arithmetic used by the computer program. For this reason, the external rays cannot be drawn in certain details of $\mathscr{M}$ with computer programs [6-8] using the double 64 bits format of the old IEEE 754-1985 Standard. This is not due to a failure of programming but a lack of accuracy of the double 64 bits format.

We are interested in the drawing of external rays of $\mathscr{M}$ [9-18], and in this paper we will analyze the possibilities of the new quadruple 128 bits format of the IEEE 754-2008 Standard. Unfortunately, as we will see in Section 5, the resolution of the quadruple format is not sufficient in some of the cases, and the same occurs with a hypothetical octuple 256 bits format (not defined yet). To avoid this problem, we will introduce in Section 4 a graphical procedure based on the escape lines [19-21] and Bézier curves [22], which allows us the drawing of the external rays of a detail of $\mathscr{M}$ when it is not possible to do it using a computer program based on the Böttcher coordinate and running with the IEEE 754 Standard.

\section{Tools to Calculate External Arguments}

2.1. Binary Expansions. As is known, there are several hyperbolic components of the same period $p$ in $\mathscr{M}$ [23]. The binary expansion of the external argument $\theta_{p}$ of an external ray landing at the root point of a period- $p$ hyperbolic component is period- $p$ periodic, and the external argument is rational with odd denominator $[2,24]$

$$
\theta_{p}=0 \cdot \overline{a_{1} a_{2} \cdots a_{p}}=\frac{a}{2^{p}-1} .
$$

In this equation $a_{1} a_{2} \cdots a_{p}$ are base ${ }_{2}$ digits such that $a_{1} a_{2} \cdots a_{p}=a$. For instance, the binary expansion of the external argument $1 / 5=3 /\left(2^{4}-1\right)$, with $a=3$ and $p=4$, is $0 . \overline{0011}=0.00110011 \cdots$, and the corresponding external ray lands at a period- 4 hyperbolic component (see Figure 1, where the external rays are drawn with a computer program using the double format of IEEE 754 [6]).

The binary expansion of the external argument $\theta_{n, m}$ of an external ray landing at a Misiurewicz point of $\mathscr{M}$, that is, a

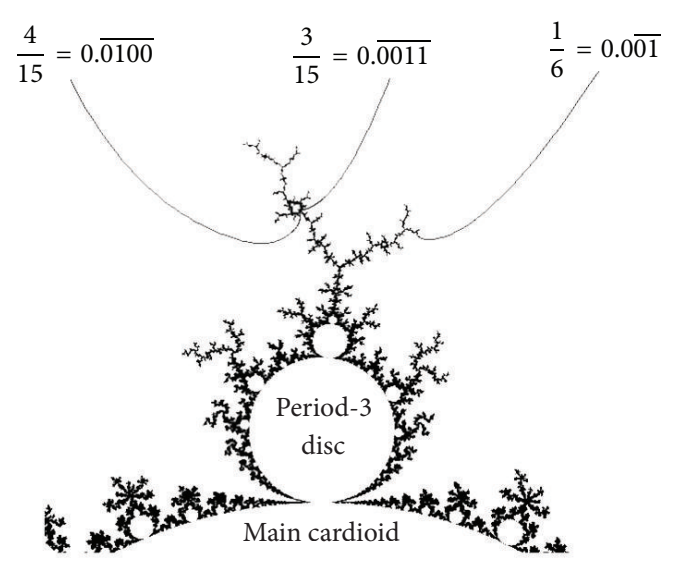

FIgURE 1: Examples of external arguments of external rays landing at a hyperbolic component and a Misiurewicz point. The figure is drawn using a computer program working with the double format of IEEE 754 Standard [6].

preperiodic point by iteration, is preperiodic of preperiod- $n$ and period- $m$. Besides, the external argument is rational with even denominator

$$
\theta_{n, m}=0 . b_{1} b_{2} \cdots b_{n} \overline{a_{1} a_{2} \cdots a_{m}}=\frac{a+b\left(2^{m}-1\right)}{2^{n}\left(2^{m}-1\right)} .
$$

Here $a_{1} a_{2} \cdots a_{m}$ and $b_{1} b_{2} \cdots b_{n}$ are base ${ }_{2}$ digits such that $a_{1} a_{2} \cdots a_{m}=a$ and $b_{1} b_{2} \cdots b_{n}=b$. For instance, the binary expansion of the external argument $1 / 6=1 / 2\left(2^{2}-1\right)$ (with $a=1, b=0, n=1$ and $m=2$ ) is $0.0 \overline{01}$ (see Figure 1).

2.2. Rotation Number. Devaney [25] associates a rational number $p / q$ to each primary disc $c$ of the Mandelbrot set (a primary disc is directly attached to the main cardioid of the set). The denominator $q$ is the period of the disc. The value of $p$ is fixed by seeing the regions, of the Julia set of $c$ when we superimpose the attracting cycle of $f_{c}(z)$ on the Julia set. The point 0 of the attracting cycle lies in the largest of these regions and the smallest is located exactly $p / q$ revolutions in the counterclockwise direction. For instance, in Figure 2 we can see that $p=2$ and $q=5$ in the $\operatorname{disc} c=-0.50+0.56 \mathrm{i}$.

We would like to note that in our papers (see, e.g., [14]) normally we write the rotation number as $q / p$ instead of $p / q$ in order to denominate the period with a $p$.

2.3. Tuning Algorithm. This algorithm is due to Douady [2]. Let $W$ be a hyperbolic component of period $p_{1}$ with centre $c_{0}$ and $W_{0}$ the main cardioid of $\mathscr{M}$ with period 1 and centre 0 . There is a continuous injection $\psi_{W}: \mathscr{M} \rightarrow$ $\mathscr{M}$ such that $c_{0}=\psi_{W}(0)$ and $W=\psi_{W}\left(W_{0}\right)$. Let us suppose the binary expansions of the external arguments of the external rays landing at the root point of $W$ are $\left(\theta_{p_{1}}^{-}, \theta_{p_{1}}^{+}\right)=\left(0 . \overline{u_{1} u_{2} \cdots u_{p_{1}}}, 0 . \overline{v_{1} v_{2} \cdots v_{p_{1}}}\right)$. Let $x$ be a landing point on $W_{0}$ of period $p_{2}$ and $x^{\prime}=\psi_{W}(x)$ the landing point on $W$ of period $p_{1} p_{2}$ that Douady called " $c_{0}$ tuned by $x$." If the binary expansions of the external arguments of the external rays landing at $x$ are $\left(\theta_{p_{2}}^{-}, \theta_{p_{2}}^{+}\right)=\left(0 . \overline{s_{1} s_{2} \cdots s_{p_{2}}}, 0 . \overline{t_{1} t_{2} \cdots t_{p_{2}}}\right)$, 


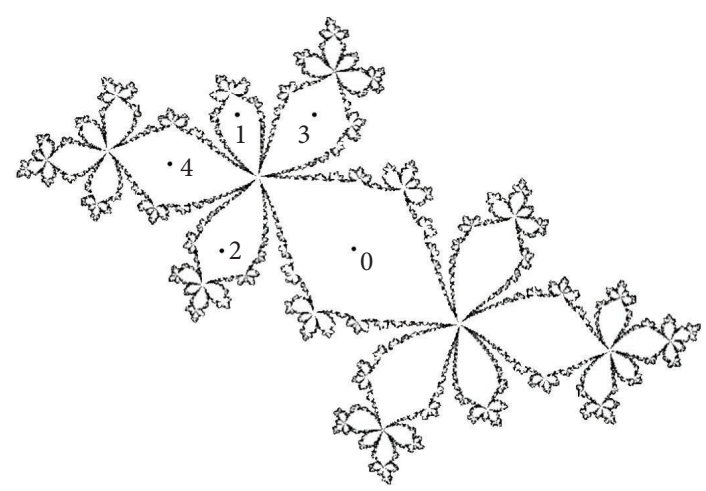

FIGURE 2: Julia set of $-0.50+0.56 \mathrm{i}$ showing the jumps in the iterative process $f_{c}(z)=z^{2}+c$.

the corresponding external rays landing at $x^{\prime}$ have external arguments whose binary expansions can be obtained by substituting each bit of $\left(\theta_{p_{2}}^{-}, \theta_{p_{2}}^{+}\right)$by $u_{1} u_{2} \cdots u_{p_{1}}$ if the bit is 0 or by $v_{1} v_{2} \cdots v_{p_{1}}$ if the bit is 1 .

For instance, let us consider the period- 4 hyperbolic component with root point located at $-0.15471+1.03104 \mathrm{i}$. The binary expansions of the external arguments of the external rays landing at this point are $\left(\theta_{4}^{-}, \theta_{4}^{+}\right)=$ $(0 . \overline{0011}, 0 . \overline{0100})$. Let $x=0.35675+0.32861 \mathrm{i}$ be the root point of a period-5 disc on the main cardioid and $\left(\theta_{5}^{-}, \theta_{5}^{+}\right)=(0 . \overline{00001}, 0 . \overline{00010})$ the binary expansions of the external arguments of the external rays landing at this point. The corresponding binary expansions of the external arguments of the rays landing at $x^{\prime}=-0.15255+1.03294 i$ are

$$
\begin{aligned}
& \left(\theta_{20}^{-}, \theta_{20}^{+}\right) \\
& \quad=(0 . \overline{00110011001100110100}, 0 . \overline{00110011001101000011}) .
\end{aligned}
$$

2.4. Narrow Hyperbolic Component. A period- $p$ hyperbolic component of the Mandelbrot set is narrow if it contains no component of equal or lesser period in its wake [26]. Then, the external arguments $\left(a^{-} /\left(2^{p}-1\right), a^{+} /\left(2^{p}-1\right)\right)$ of the external rays landing at the root point of a period- $p$ narrow hyperbolic component differ by $1 /\left(2^{p}-1\right)$, because $a^{+}-a^{-}=1$. For instance, the binary expansions $\left(\theta_{4}^{-}, \theta_{4}^{+}\right)=(0 . \overline{0011}, 0 . \overline{0100})$, see Figure 1, correspond to a narrow period-4 hyperbolic component.

It is obvious that we need a computer program with precision prec $=2^{- \text {res }}$ better than $1 /\left(2^{p}-1\right) \simeq 2^{-p}$ to be able to draw separately the two external rays of a period- $p$ narrow hyperbolic component near its landing point. Taking into account the resolutions of the formats of the IEEE 7542008 Standard (see Table 1), the drawing of the two external rays of a narrow hyperbolic component is impossible when its period is greater than 23,52 , or 112 , and we use a single, double or quadruple format, respectively.

2.5. Schleicher's Algorithm. Schleicher's algorithm allows us to find the binary expansions of the external arguments of the two external rays landing at the largest disc between two given with rotation numbers $p_{1} / q_{1}$ and $p_{2} / q_{2}$, when the binary expansions of the external rays landing at these discs are known (a detailed description of the algorithm can be seen in [27]).

First, we determine the rotation number of the largest disc by Farey addition [28] that is, $\left(p_{1} / q_{1}\right) \oplus\left(p_{2} / q_{2}\right)=\left(p_{1}+\right.$ $\left.p_{2}\right) /\left(q_{1}+q_{2}\right)$. Second, we assume that the binary expansions of the two external rays closest to the $\left(p_{1}+p_{2}\right) /\left(q_{1}+q_{2}\right)$ disc are known, and they are $0 . \overline{u_{1} u_{2} \cdots u_{q_{1}}}$ and $0 . \overline{v_{1} v_{2} \cdots v_{q_{2}}}$ such that $0 . \overline{u_{1} u_{2} \cdots u_{q_{1}}}<0 . \overline{v_{1} v_{2} \cdots v_{q_{2}}}$. Third, the binary expansions of the external rays landing at the $\left(p_{1}+p_{2}\right) /\left(q_{1}+q_{2}\right)$ disc are $\left(0 . \overline{u_{1} u_{2} \cdots u_{q_{1}} v_{1} v_{2} \cdots v_{q_{2}}}, 0 . \overline{v_{1} v_{2} \cdots v_{q_{2}} u_{1} u_{2} \cdots u_{q_{1}}}\right)$.

For instance, the higher external argument of the disc with rotation number $1 / 3$ is $0 . \overline{010}$, and the smaller external argument of the disc with rotation number $2 / 5$ is $0 . \overline{01001}$. The biggest disc between the two former ones has rotation number $1 / 3 \oplus 2 / 5=3 / 8$ and external arguments $(0 . \overline{01001001}, 0 . \overline{01001010})$.

2.6. Binary Expansions in Multiple-Spiral Medallions. As is known, $\mathscr{M}$ contains small copies of itself (babies Mandelbrot sets (BMS)) which in turn contain smaller copies of $\mathscr{M}$, and so on ad infinitum. But the $\mathscr{M}$ set is not self-similar. Actually, every BMS has its own pattern of external decorations. Some of these decorations are called cauliflowers [29], embedded Julia sets [30], or multiple-spiral medallions [11].

Using the symbolic binary expansion, it has been conjectured [11] that the pair of binary expansions of the external arguments of the external rays landing at the cusp of the cardioid of the central BMS in a multiple-spiral medallion $\left(0 . \overline{b_{1}}, 0 . \overline{b_{2}}\right)$ can be written starting from the binary expansions of its parent $\left(0 . \overline{p_{1}}, 0 . \overline{p_{2}}\right)$ and its gene $\left(0 . \overline{g_{1}}, 0 . \overline{g_{2}}\right)$ in the form $0 . \overline{b_{*}}=0 . \overline{p_{*} p_{*} \cdots p_{*} g_{*}}$ (where $b_{*}$ is $b_{1}$ or $b_{2}, p_{*}$ is $p_{1}$ or $p_{2}$ and $g_{*}$ is $g_{1}$ or $\left.g_{2}\right)$. The binary expansions $\left(0 . \overline{\beta_{1}}, 0 . \overline{\beta_{2}}\right)$ of a noncentral BMS have the form $0 . \overline{\beta_{*}}=0 . \overline{b_{*}} \underbrace{p_{*} p_{*} \cdots p_{*}}_{j}$ (where $\beta_{*}$ is $\beta_{1}$ or $\beta_{2}$ and $j$ is the level of the BMS [15]). In Figure 3 we can see examples of multiple-spiral medallions, where the BMSs until the third level are shown.

\section{The End Point of the Drawing of an External Ray}

When we draw an external ray by means of a computer program using the Böttcher coordinate, we observe that the drawing of the ray is interrupted when it comes close to the landing point; that is, the drawing of an external ray has an end point. This limitation is due to a lack of resolution of the drawing program that usually works with the floating-point arithmetic of the IEEE 754 Standard.

As an example, in Figure 4 we can see the drawing of the external ray $\theta_{3}^{-}=1 / 7=0 . \overline{001}$ near the tangent point of the disc of rotation number $1 / 3$ with the main cardioid, starting from the programs of Chéritat [6], Kawahira [7], and Jung [8]. As far as we know, these programs use the double format of the IEEE 754 Standard with res double $=52$. The end point 


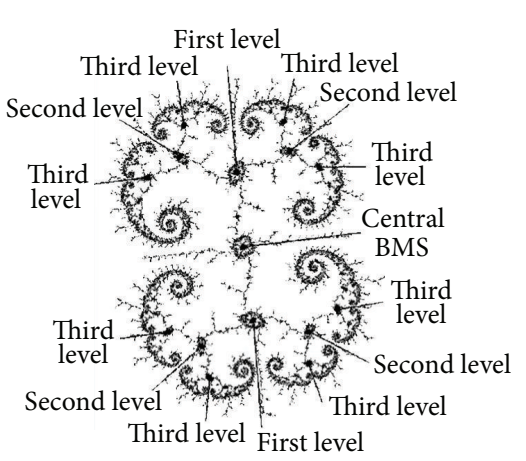

(a)

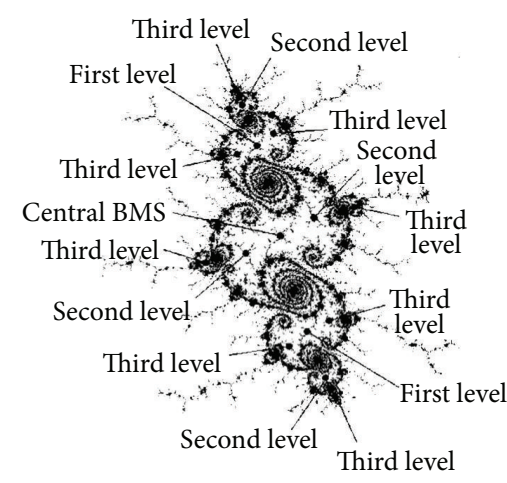

(b)

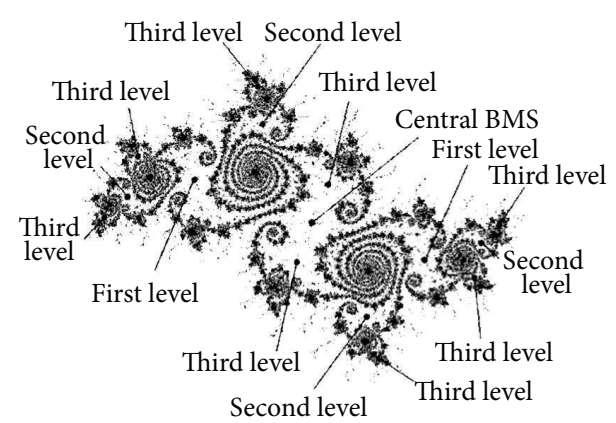

(c)

FIGURE 3: Examples of multiple-spiral medallions [13] in the Mandelbrot set showing the first, second, and third levels of BMSs. (a) Singlespiral medallion $-0.1543869+1.0308295 i$. (b) Double-spiral medallion $-0.16111880+1.03632203 i$. (c) Triple-spiral medallion $-0.15403780+$ $1.03692215 i$.

of the drawing of the external ray $0 . \overline{001}$ is approximately at $-0.088+0.652 \mathrm{i}$, beneath the period-51 disc. Therefore, we can assume that the end point of the drawing of an external ray occurs when the discs of its vicinity have periods about the number of bits of the mantissa of the format of the IEEE 754.

Let us consider the drawing of two close external rays $\theta_{x}$ and $\theta_{y}$ landing at points $x$ and $y$ (see Figure 5). If the program has res $=\infty$ as in (a), away from points $x$ and $y$, the two external rays go together in the same pixels until the graphical bifurcation point g.b.p. where they begin to separate. Finally, the landing points $x$ and $y$ would be reached. However, when we use a program with an IEEE 754 format, with finite resolution, the landing points cannot be reached, and there are two cases. When $\left|\theta_{x}-\theta_{y}\right|>2^{- \text {res }}$, see (b), the end points appear after reaching the g.b.p. When $\left|\theta_{x}-\theta_{y}\right|<2^{- \text {res }}$, see (c), the end points appear before reaching the g.b.p.

\section{Drawing External Rays When the Resolution of the IEEE 754 Is Not Sufficient}

When the number of bits of the period (or the sum of the number of bits of the preperiod and period) of the binary expansion of the external argument of an external ray is greater than the number of bits of the quadruple format of the IEEE 754 Standard, the drawing of the external ray near the landing point by means of a computer program running with this Standard is obviously impossible. In this case we operate as follows (see Figure 6, where we obtain the same external rays that in Figure 1).

First, we draw the detail of the Mandelbrot set with escape lines (blue colour in Figure 6) [19-21]. The equation of an escape line is $\bmod \left[f_{c}^{n}(c)\right]=r_{e}$, where $r_{e} \geq 2$ is the escape radius and $f_{c}^{0}=c, f_{c}^{1}=c^{2}+c, f_{c}^{2}=\left(c^{2}+c\right)^{2}+c \cdots$. For $n=0,1,2 \ldots$ we have the different escape lines. The escape lines are closed curves that have a clear physical meaning: a point $c$ out of the escape line $n$ but inside the escape line $n-1$ need $n$ iterations to leave the circle of radius $r_{e}$ (i.e., to escape to infinity). Hence, the escape line $\infty$ is the boundary of the Mandelbrot set. As is well known, if the escape radius is large compared to the size of the set (e.g., $r_{e}=10^{5}$ ) the escape lines can be considered as equipotential lines. The escape lines are obtained as a consequence of the iteration process in the drawing of the detail of the Mandelbrot set, and they do not need to be obtained with the Böttcher coordinate.

Second, we draw manually the external rays by means of Bézier curves (red colour in Figure 6) [22] starting from the landing points of the external rays in such a way that they are perpendicular to the escape lines.

\section{The Drawing of the External Rays Requires Great Computer Resolution}

Figure 7 shows a region of the Mandelbrot set near the tangent point of the disc of rotation number $1 / 3$ with the main cardioid. Between the abscises -0.1184 and -0.1178 there are 1163 pixels, and therefore the distance between two consecutive pixels is $d=(0.1184-0.1178) / 1163=5.1 \times$ $10^{-7}$. The figure has been drawn by means of a program using the IEEE 754 double format where $2^{- \text {res }_{\text {double }}}=2^{-52} \simeq$ $2.2 \times 10^{-16} \ll d$, with escape lines (in blue colour) and Bézier curves (in red colour), according to Section 4. As we can see next, it is not possible to draw the external rays in this figure by means of a computer program using the Bötcher coordinate, due to a lack of resolution of the quadruple format of the IEEE 754 Standard.

In the upper part of Figure 7, there are discs with periods $3 \times 84,3 \times 85, \ldots, 3 \times 91$, attached to the disc $1 / 3$. For example, the external arguments of the external rays landing at the tangent point of the disc with period $3 \times 89=$ 267 can be obtained by tuning, and they are $\left(\theta_{267}^{-}, \theta_{267}^{+}\right)=$ $\left(0 . \overline{001}^{88} 010,0 . \overline{\overline{001}^{87} 010001}\right)$. Note that the first 262 digits of $\theta_{267}^{-}$and $\theta_{267}^{+}$are the same. Therefore, to be able to draw these external rays separately near the landing point, the resolution of the computer program must be greater than 262. Obviously, this resolution exceeds the possibilities of the quadruple format (128 bits) of the IEEE 754 Standard. 


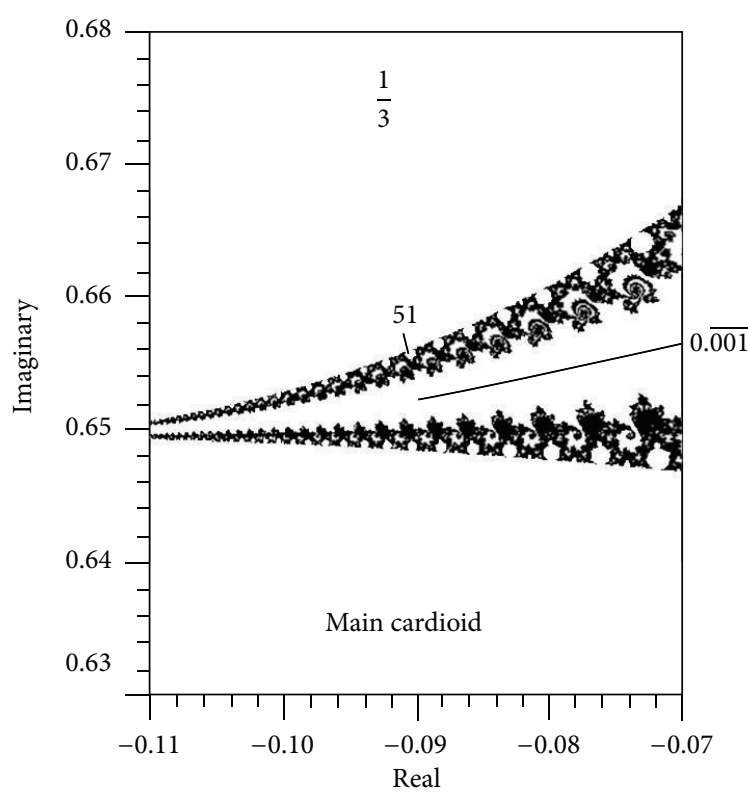

(a)

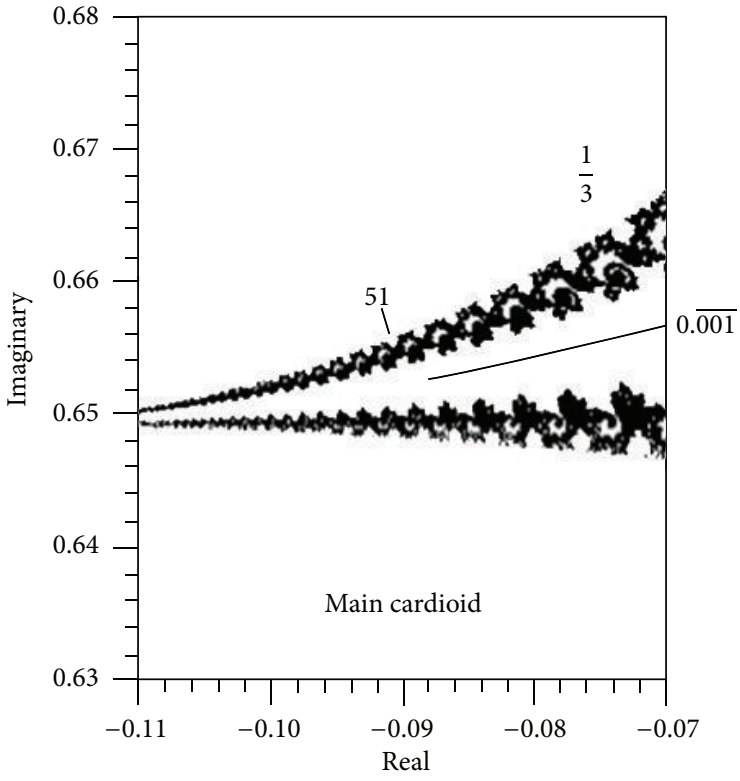

(b)

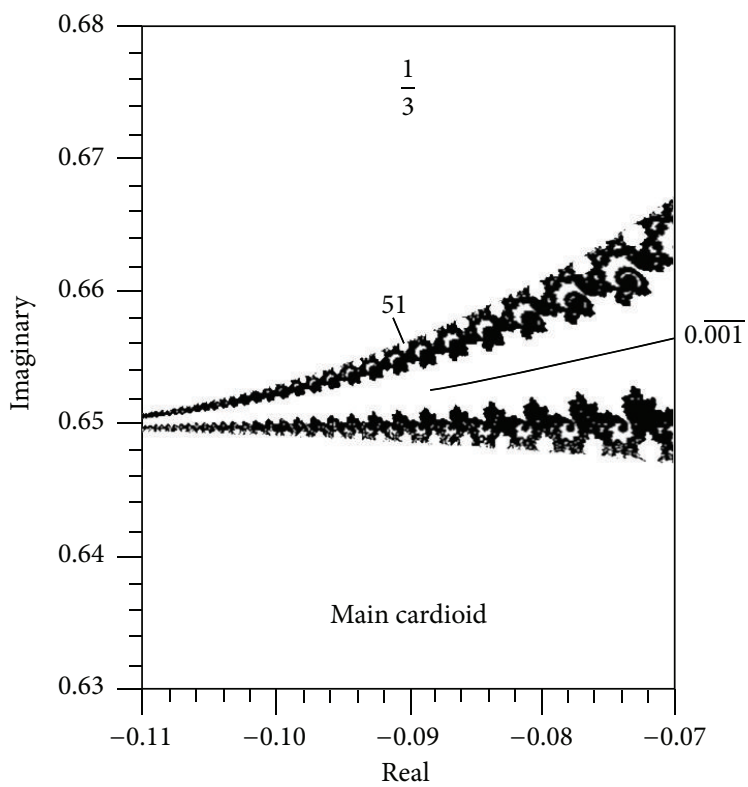

(c)

FIGURE 4: End point of the drawing of the external ray $0 . \overline{001}$ obtained with three different programs working with the double format of IEEE 754 Standard. (a) Chéritat's program [6]. (b) Kawahira’s program [7]. (c) Jung's program [8].

In the lower part of Figure 7 there are discs with periods $3 \times 83+4,3 \times 84+4, \ldots, 3 \times 90+4$ attached to the main cardioid. The external arguments of the external rays landing at the tangent point of the disc with period $3 \times 88+4=$ 268 can be obtained by the Schleicher's algorithm, and they are $\left(\theta_{268}^{-}, \theta_{268}^{+}\right)=\left(0 . \overline{\overline{001}}^{88} 0001,0 . \overline{\overline{001}}^{88} 0010\right)$. Note that this disc is narrow. The first 266 digits of $\theta_{268}^{-}$and $\theta_{268}^{+}$are the same, and, again, the external rays $\theta_{268}^{-}$and $\theta_{268}^{+}$of Figure 7 cannot be drawn separately with a computer program using the quadruple format (neither with the octuple format that is not defined yet).

Taking into account that $\theta_{267}^{+}>\theta_{267}^{-}>\theta_{3}^{-}>\theta_{268}^{+}>\theta_{268}^{-}$, the external ray $\theta_{3}^{-}$is between rays that cannot be drawn. We conclude that the external ray $\theta_{3}^{-}$of Figure 7 cannot be drawn by a computer program using the quadruple format of IEEE 754. In this case, we propose the drawing of the external rays according to Section 4 . 


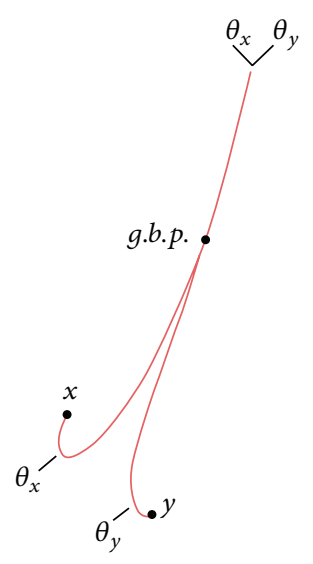

(a)

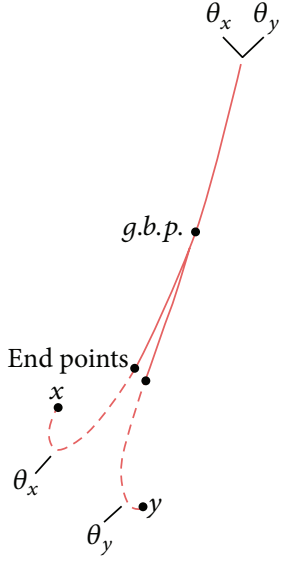

(b)

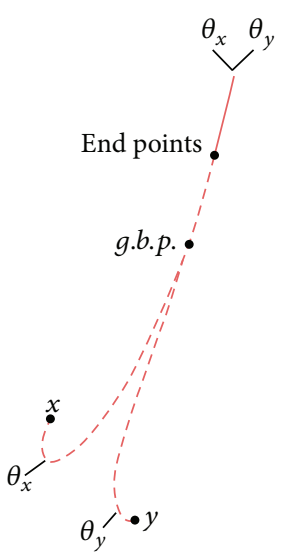

(c)

FIgure 5: The drawing of two external rays $\theta_{x}$ and $\theta_{y}$ when the difference $\left|\theta_{x}-\theta_{y}\right|$ is very small. (a) Ideal case res $=\infty$. (b) Practical case when $\left|\theta_{x}-\theta_{y}\right|>2^{- \text {res }}$. (c) Practical case when $\left|\theta_{x}-\theta_{y}\right|<2^{- \text {res }}$.

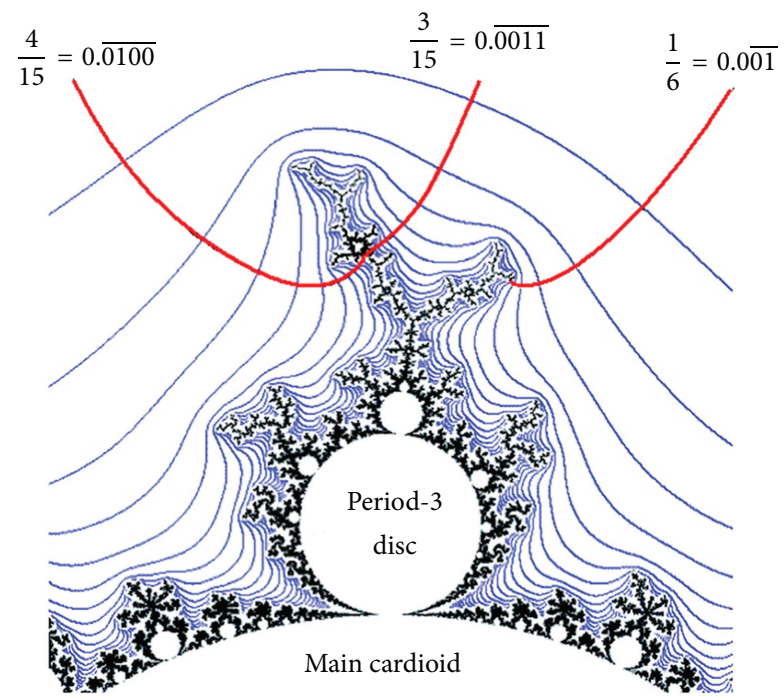

Figure 6: Reproduction of Figure 1 according to Section 4.

We have shown an example where the double format of IEEE 754 Standard can draw a detail of the Mandelbrot set but the quadruple format (with more precision) cannot draw the external rays in the detail. Hence, the drawing of the external rays in a region of the Mandelbrot set needs greater computer resolution than the drawing of the detail itself.

5.1. Example. Let us consider the double-spiral medallion $[13,15]$ located at $-0.16111880+1.03632203 i$. Figure 8 shows the location of the medallion, and in Figure 8(a) we can see his parent $\left(0 . \overline{p_{1}}, 0 . \overline{p_{2}}\right)=(0 . \overline{0011}, 0 . \overline{0100})$ and his gene $\left(0 . \overline{g_{1}}, 0 . \overline{g_{2}}\right)=(0 . \overline{001}, 0 . \overline{010})[9,11]$.

Figure 9 shows the medallion in detail with escape lines (in blue colour) and external rays (in red colour) according to Section 4. As is known [15], the binary expansions of the external arguments of the external rays landing at the cusp of the central BMS of a double-spiral medallion have symbolic binary expansions $\left(0 . \overline{b_{1}}, 0 . \overline{b_{2}}\right)=$ $\left(0 .{\overline{p_{1} p_{2}}}^{i} p_{1} p_{1} g_{2}, 0 . \overline{{\overline{p_{1} p_{2}}}^{i+1} g_{1}}\right)$ with $i=1,2,3 \ldots$ In the medallion of Figure 9 we have $i=32$ and

$$
\begin{aligned}
& \left(0 . \overline{b_{1}}, 0 . \overline{b_{2}}\right) \\
& =\left(0 . \overline{\overline{00110100}^{32} 00110011010}, 0 . \overline{\overline{00110100}^{33} 001}\right) .
\end{aligned}
$$

Therefore the period of the central BMS is 267 , as we easily can verify by direct iteration of $-0.16111880+1.03632203 \mathrm{i}$. Note that the first 261 bits of the pair $\left(0 . \overline{b_{1}}, 0 . \overline{b_{2}}\right)$ are the same.

The medallion has two principal tips. One of them can be obtained as the limit of the BMSs $\left(0 . \overline{b_{1} p_{2}}, 0 . \overline{b_{2} p_{1}}\right)$, $\left(0 . \overline{b_{1} p_{2} p_{2}}, 0 . \overline{b_{2} p_{1} p_{1}}\right),\left(0 . \overline{b_{1} p_{2} p_{2} p_{2}}, 0 . \overline{b_{2} p_{1} p_{1} p_{1}}\right) \ldots$, and it is

$\left(0 . b_{1} \overline{p_{2}}, 0 . b_{2} \overline{p_{1}}\right)$

$$
=\left(0 . \overline{00110100}^{32} 00110011010 \overline{0100}, 0 . \overline{00110100}^{33} 001 \overline{0011}\right) \text {. }
$$

Analogously, the other principal tip is

$$
\begin{aligned}
& \left(0 . b_{1} \overline{p_{1}}, 0 . b_{2} \overline{p_{2}}\right) \\
& =\left(0 . \overline{00110100}^{32} 00110011010 \overline{0011}, 0 . \overline{00110100}^{33} 001 \overline{0100}\right) .
\end{aligned}
$$

The two tips have preperiods 267 and periods 4 . Also note that the first 261 bits of the pairs $\left(0 . b_{1} \overline{p_{2}}, 0 . b_{2} \overline{p_{1}}\right)$ and $\left(0 . b_{1} \overline{p_{1}}, 0 . b_{2} \overline{p_{2}}\right)$ are the same.

It is evident that each one of the binary expansions of the external arguments of the BMSs and Misiurewicz points in this medallion must be in the interval $\left[0 . b_{1} \overline{p_{1}}, 0 . b_{2} \overline{p_{2}}\right]$ where the first 261 bits of the binary expansions are the same. We deduce that it is not possible to draw any of the external rays inside of this medallion using a computer program working 


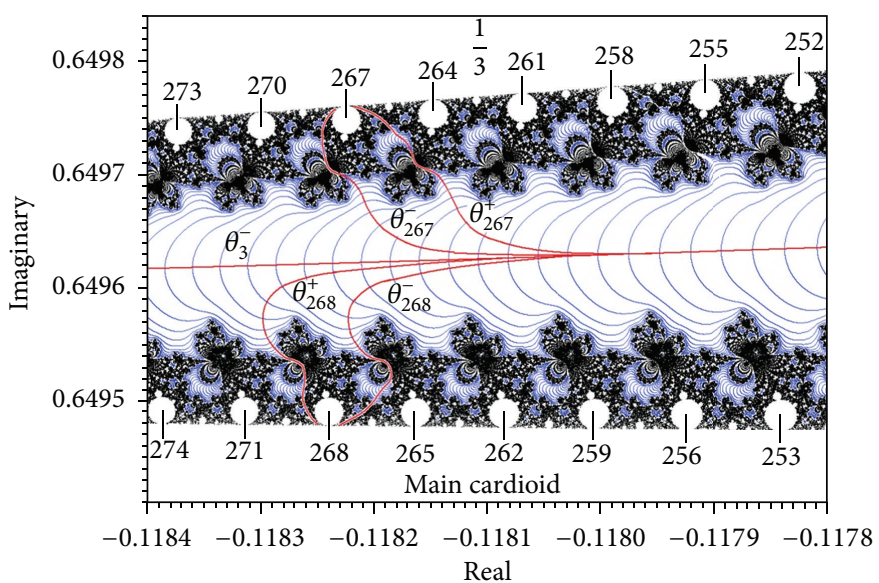

FIGURE 7: Detail of the Mandelbrot set near the tangent point of the disc with rotation number 1/3.

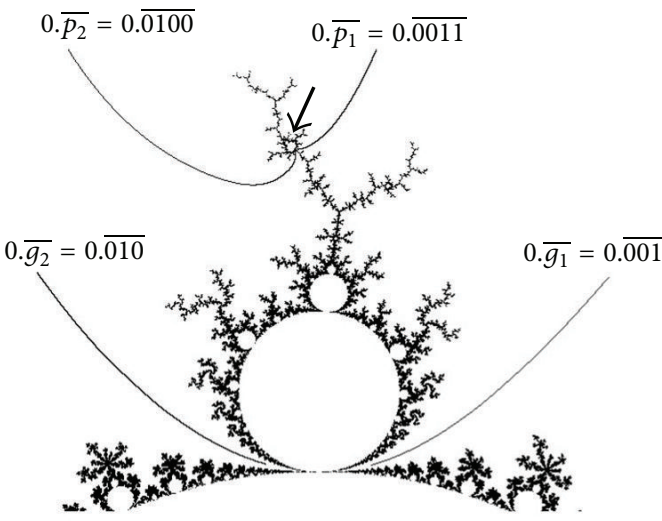

(a)

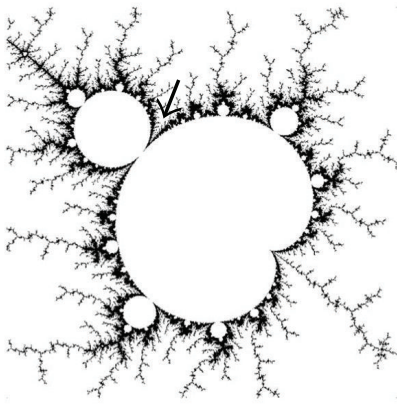

(b)

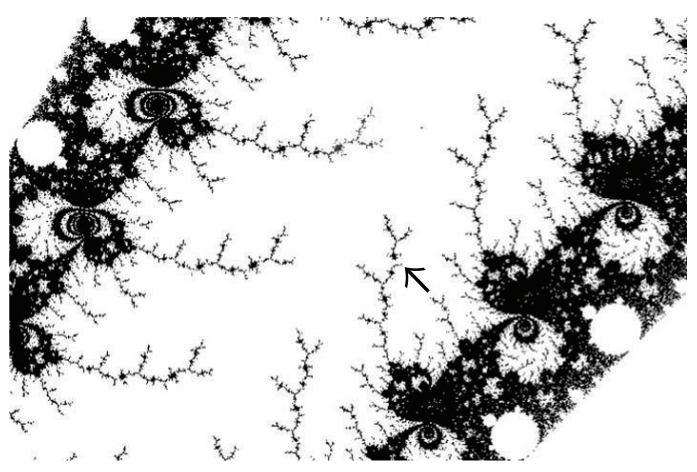

(c)

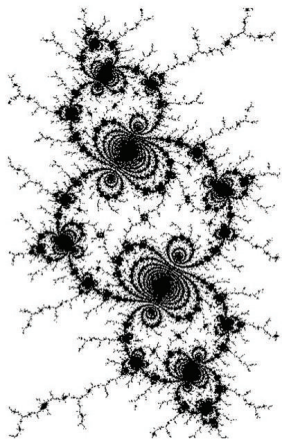

(d)

FIGURE 8: Successive magnifications for locating the double-spiral medallion centred at $-0.16111880+1.03632203 \mathrm{i}$ (the medallion is marked by the arrow). (a) The parent $\left(0 . \overline{p_{1}}, 0 . \overline{p_{2}}\right)=(0 . \overline{0011}, 0 . \overline{0100})$ in the wake of the gene $\left(0 . \overline{g_{1}}, 0 . \overline{g_{2}}\right)=(0 . \overline{001}, 0 . \overline{010})$. (b) The period-4 parent. (c) The valley between the period- $(2 \times 4)$ disc of the parent and the cardioid of the parent. (d) The medallion.

with the quadruple format (128 bits) of IEEE 754 Standard, nor with the octuple format (256 bits, not yet defined).

However it is possible to draw the external rays of the medallion according to Section 4 . In Figure 9 we can see the external rays landing at the central BMS $\left(0 . \overline{b_{1}}, 0 . \overline{b_{2}}\right)$, the tips $\left(0 . b_{1} \overline{p_{2}}, 0 . b_{2} \overline{p_{1}}\right)$ and $\left(0 . b_{1} \overline{p_{1}}, 0 . b_{2} \overline{p_{2}}\right)$, the two BMSs of the first level, the four BMSs of the second level, and the eight BMSs of the third level.

\section{Conclusions}

It has been shown that the drawing of the external rays in a detail of the Mandelbrot set, using a computer program starting from the Böttcher coordinate and running with the current IEEE 754 Standard for Floating-Point Arithmetic, requires more resolution than the drawing of the detail itself. For this cause, in certain details of the Mandelbrot set 


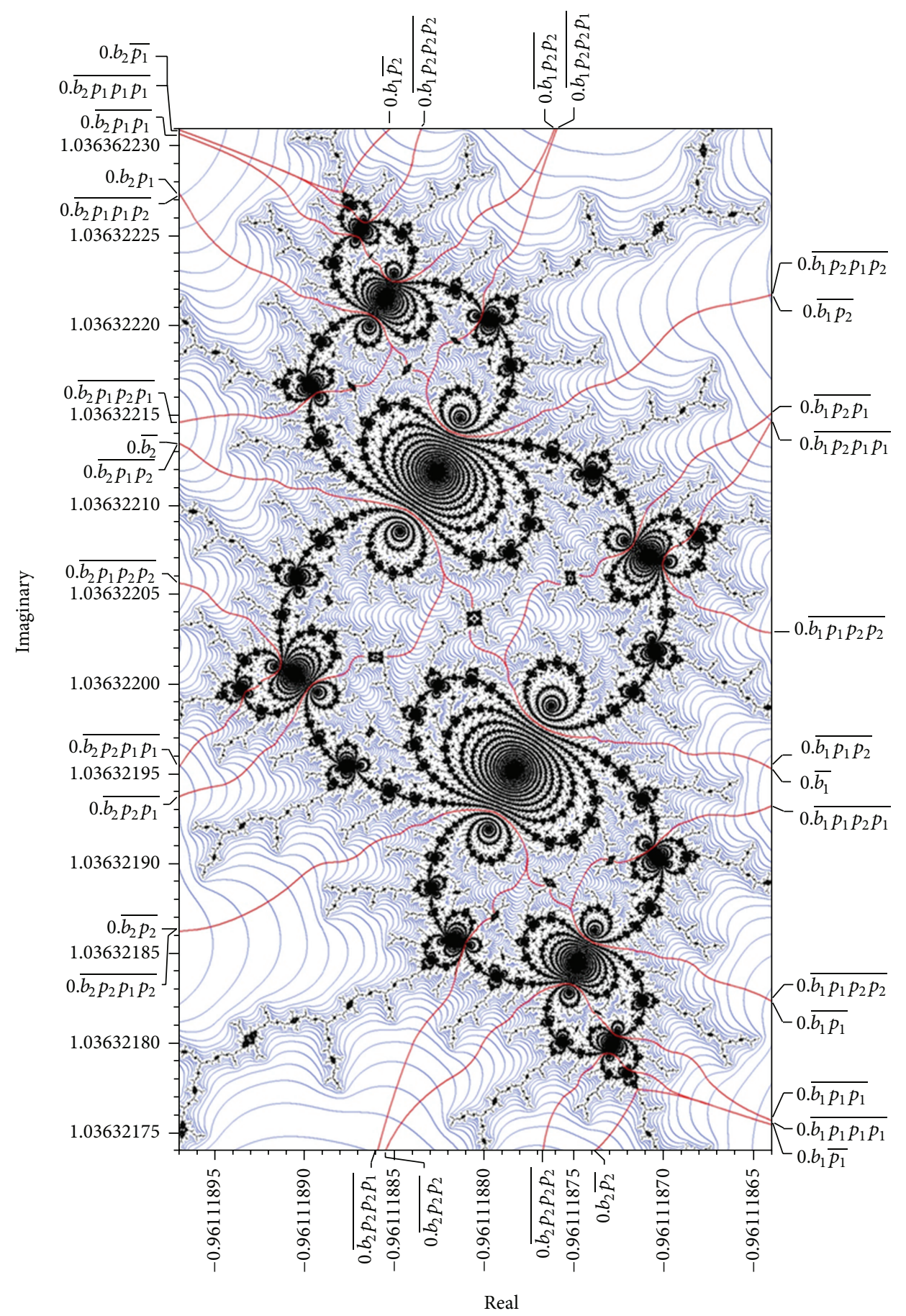

Figure 9: Double-spiral medallion located at $-0.16111880+1.03632203 i$ showing escape lines (blue colour) and Bézier curves (red colour) according to Section 4 .

obtained with the quadruple format of this Standard (the most precise), it is not possible to draw the external rays due to a lack of resolution. In these cases we have introduced a method based on escape lines and Bézier curves, which allows the drawing of the external rays.

\section{Acknowledgment}

This work has been partially supported by Ministerio de Ciencia e Innovación (Spain) under the Grant no. TIN201122668. 


\section{References}

[1] A. Douady and J. H. Hubbard, "Itération des polynômes quadratiques complexes," Comptes Rendus des Séances de l'Académie des Sciences. Série I. Mathématique, vol. 294, no. 3, pp. 123-126, 1982.

[2] A. Douady, "Algorithms for computing angles in the Mandelbrot set," in Chaotic Dynamics and Fractals, M. Barnsley and S. G. Demko, Eds., vol. 2, pp. 155-168, Academic Press, New York, NY, USA, 1986.

[3] A. Douady, "Julia set and the Mandelbrot set," in The Beauty of Fractals, H. O. Peitgen and P. H. Richter, Eds., pp. 161-173, Springer, Berlin, Germany, 1986.

[4] L. Carleson and T. W. Gamelin, Complex Dynamics, Springer, Berlin, Germany, 1993.

[5] IEEE 754-2008 Standard for Floating-Point Arithmetic, http:// ieeexplore.ieee.org/xpl/freeabs_all.jsp?arnumber=4610935.

[6] A. Chéritat, "Dynamique Holomorphe," 1999, http://www.math .univ-toulouse.fr/ cheritat/.

[7] T. Kawahira, "Mandel," 2008, http://www.math.nagoya-u.ac.jp/ $\sim$ kawahira/programs/mandel.html.

[8] W. Jung, "Mandel: software for real and complex dynamics," 2012, http://www.mndynamics.com/indexp.html.

[9] M. Romera, G. Pastor, G. Álvarez, and F. Montoya, "External arguments of Douady cauliflowers in the Mandelbrot set," Computers and Graphics, vol. 28, no. 3, pp. 437-449, 2004.

[10] G. Pastor, M. Romera, G. Álvarez, and F. Montoya, "Operating with external arguments in the Mandelbrot set antenna," Physica D, vol. 171, no. 1-2, pp. 52-71, 2002.

[11] M. Romera, G. Pastor, G. Álvarez, and F. Montoya, "External arguments in the multiple-spiral medallions of the Mandelbrot set," Computers and Graphics, vol. 30, no. 3, pp. 461-470, 2006.

[12] G. Pastor, M. Romera, G. Álvarez, and F. Montoya, "External arguments for the chaotic bands calculation in the Mandelbrot set," Physica A, vol. 353, no. 1-4, pp. 145-158, 2005.

[13] M. Romera, G. Álvarez, D. Arroyo, A. B. Orue, V. Fernandez, and G. Pastor, "Drawing and computing external rays in the multiple-spiral medallions of the Mandelbrot set," Computers and Graphics, vol. 32, no. 5, pp. 597-610, 2008.

[14] G. Pastor, M. Romera, G. Álvarez, D. Arroyo, and F. Montoya, "Equivalence between subshrubs and chaotic bands in the Mandelbrot set," Discrete Dynamics in Nature and Society, vol. 2006, Article ID 70471, 25 pages, 2006.

[15] M. Romera, G. Pastor, A. B. Orue, D. Arroyo, and F. Montoya, "Coupling patterns of external arguments in the multiple-spiral medallions of the Mandelbrot set," Discrete Dynamics in Nature and Society, vol. 2009, Article ID 135637, 14 pages, 2009.

[16] G. Pastor, M. Romera, G. Álvarez, J. Nunez, D. Arroyo, and F. Montoya, "Operating with external arguments of Douady and Hubbard," Discrete Dynamics in Nature and Society, vol. 2007, Article ID 45920, 17 pages, 2007.

[17] G. Pastor, M. Romera, G. Álvarez et al., "Algorithm for external arguments calculation of the nodes of a shrub in the Mandelbrot set," Fractals, vol. 16, no. 2, pp. 159-168, 2008.

[18] G. Pastor, M. Romera, G. Álvarez et al., "A general view of pseudoharmonics and pseudoantiharmonics to calculate external arguments of Douady and Hubbard," Applied Mathematics and Computation, vol. 213, no. 2, pp. 484-497, 2009.

[19] H.-O. Peitgen and P. H. Richter, The Beauty of Fractals, Springer, New York, NY, USA, 1986.
[20] K. W. Philip, "Field lines in the Mandelbrot set," Computers and Graphics, vol. 16, no. 4, pp. 443-447, 1992.

[21] M. Romera, G. Pastor, and F. Montoya, "Graphic tools to analyse one-dimensional quadratic maps," Computers and Graphics, vol. 20, no. 2, pp. 333-339, 1996.

[22] P. Bézier, "Procédé de définition numérique des courbes et surfaces non mathématiques: système Unisurf," Automatisme, vol. 13, pp. 189-196, 1968.

[23] M. Lutzky, "Counting stable cycles in unimodal iterations," Physics Letters A, vol. 131, no. 4-5, pp. 248-250, 1988.

[24] B. Branner, "The Mandelbrot set," in Chaos and Fractals: The Mathematics Behind the Computer Graphics, vol. 39 of Proceedings of Symposia in Applied Mathematics, pp. 75-105, American Mathematical Society, Providence, RI, USA, 1989.

[25] R. L. Devaney, "The fractal geometry of the Mandelbrot set. 2. How to count and how to add," Fractals, vol. 3, no. 4, pp. 629640, 1995.

[26] D. Schleicher, "Internal addresses of the Mandelbrot set and Galois groups of polynomials," http://arxiv.org/abs/math/ 9411238.

[27] R. L. Devaney, "The complex dynamics of quadratic polynomials," in Complex Dynamical Systems, R. L. Devaney, Ed., vol. 49 of Proceedings of Symposia in Applied Mathematics, pp. 1-29, American Mathematical Society, Cincinnati, Ohio, USA, 1994.

[28] J. Farey, "On a curious property of vulgar fractions," Philosophical Magazine, vol. 47, pp. 385-386, 1816.

[29] A. Douady, X. Buff, R. L. Devaney, and P. Sentenac, "Baby Mandelbrot sets are born in cauliflowers," in The Mandelbrot Set, Theme and Variations, T. Lei, Ed., vol. 274, pp. 19-36, University Press Cambridge, 2000.

[30] R. P. Munafo, "Embedded Julia set," 2008, http://mrob.com/ $\mathrm{pub} /$ muency/embeddedjuliaset.html. 


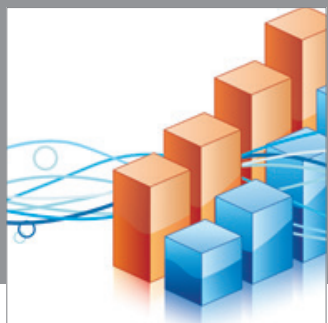

Advances in

Operations Research

mansans

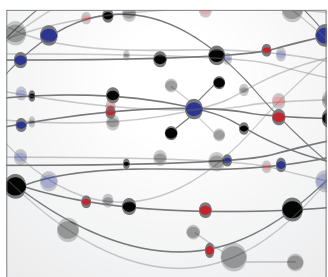

The Scientific World Journal
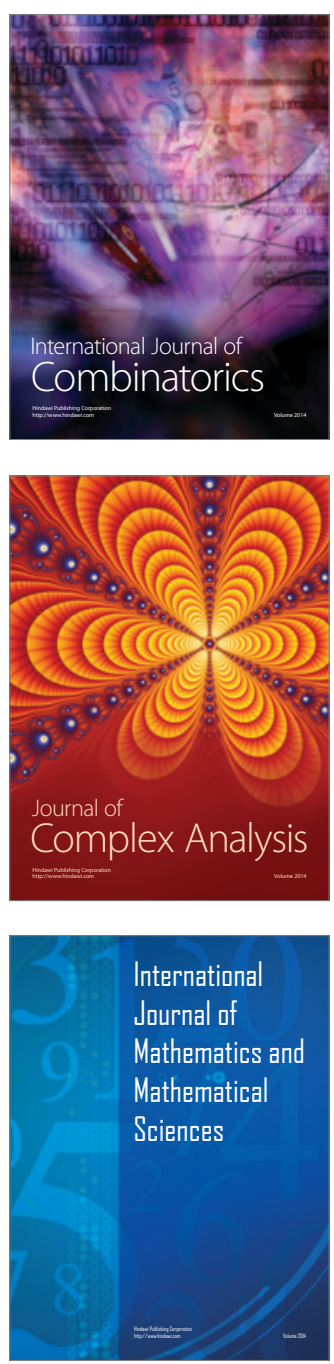
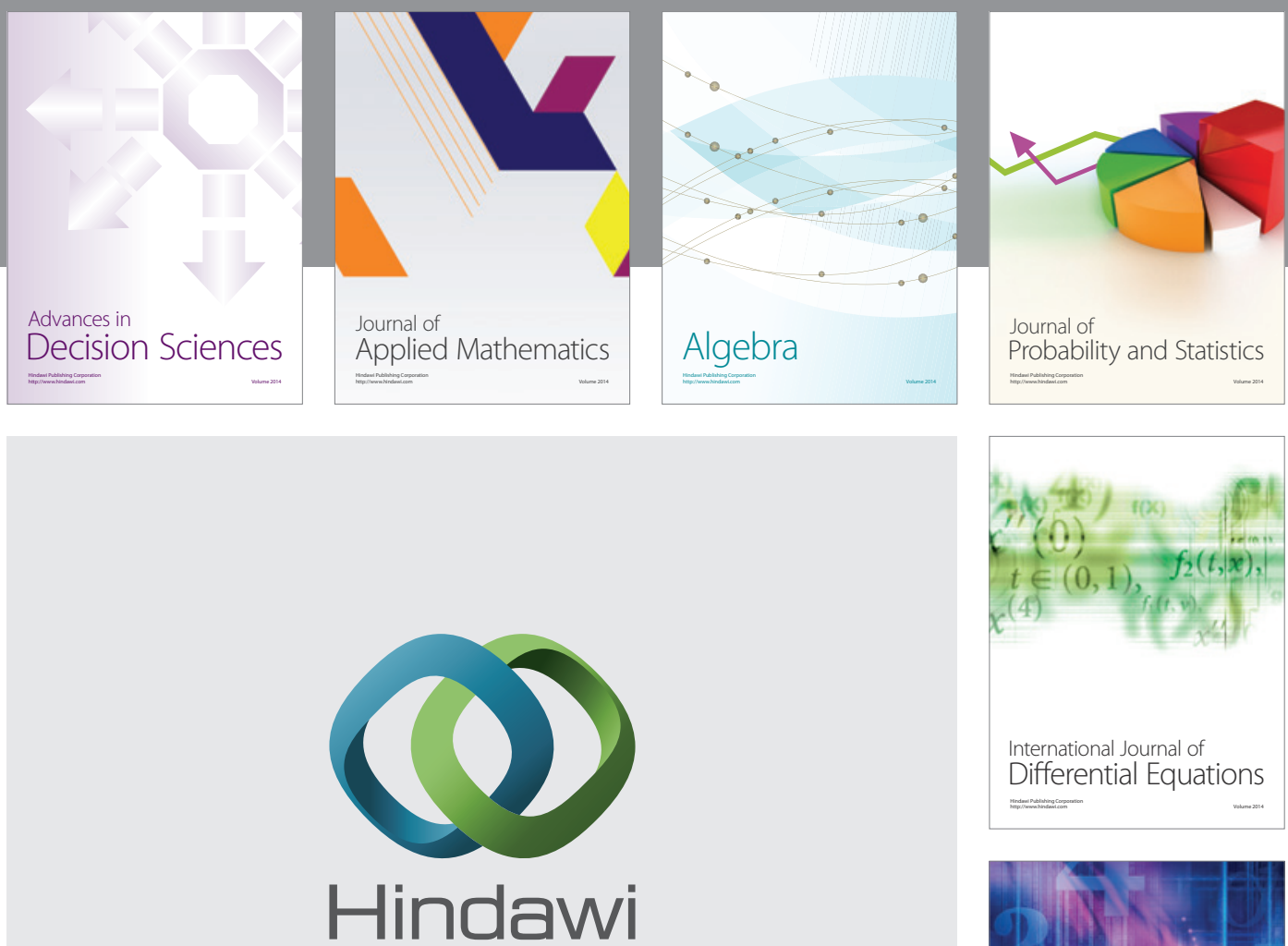

Submit your manuscripts at http://www.hindawi.com
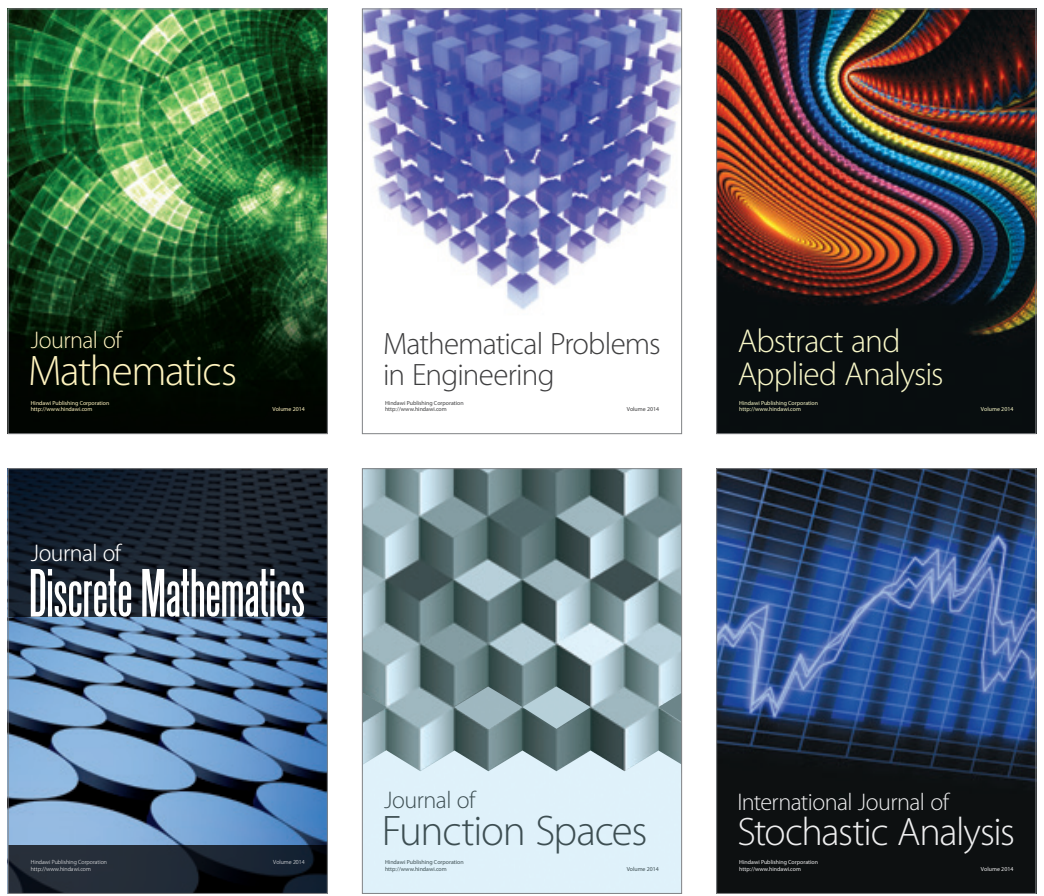

Journal of

Function Spaces

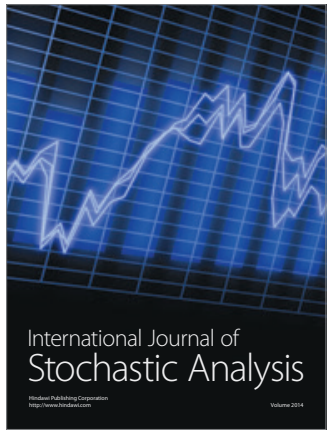

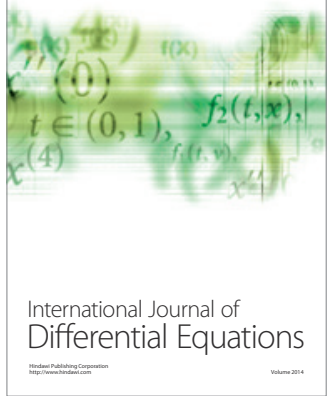
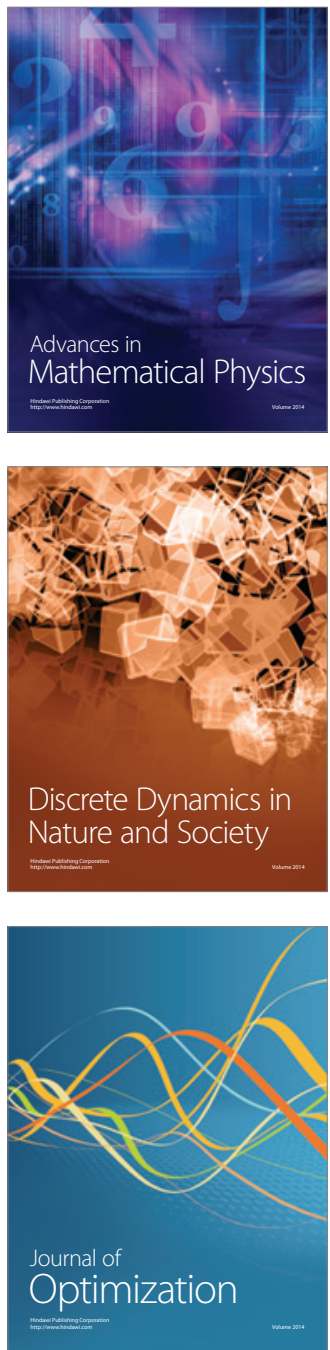